\title{
Incidencia de la actividad minera en el desarrollo social de la parroquia Chumblín del cantón San Fernando
}

\section{Incidence of the mining activity on the social development in "Chumblin, San Fernando"}

Leticia Chuñir Panjón. ${ }^{1}$, Daniele Covri Rivera. ${ }^{2} \&$ Yonimiler Castillo Ortega. ${ }^{3}$

Recibido: 29-01-2021 / Revisado: 03-02-2021 /Aceptado: 28-02-2021/ Publicado: 05-03-2021

\begin{abstract}
.
DOI: https://doi.org/10.33262/concienciadigital.v4i1.2.1608

Introduction: Mining is not a new activity, since pre-Hispanic times there is evidence of artisanal mining where the importance of conserving the environment was not considered, as time goes by, this has improved until reaching large-scale mining processes where, for the extraction of the mineral, very demanding parameters that guarantee the good living of human beings have to be met; that is, sustainable and sustainable mining has to be practiced. Nowadays, mining activity is a relevant aspect within the country, since it is considered as emblematic and strategic within the economy and development not only locally but also at a national level. Objective: This research will determine how the Loma Larga mining project affects the social development of the Chumblín parish. This research will determine how the Loma Larga mining project affects the social development of the Chumblín parish. Methodology and Results: The research is based fundamentally on a documentary analysis; in the first instance of the Loma Larga mining project under the concession of INV Minerales; then a study is made of the main indicators of the Chumblín parish, population, education level, aging index, youth, dependency, NBI, PET, PEA, PEI, economic activity, and access to basic services that allow us to establish a current diagnosis of the study area to continue with an investigation of the social and

\footnotetext{
${ }^{1}$ Universidad Católica de Cuenca, Posgrado, Maestría en Desarrollo Local Mención en Ordenamiento Territorial, Cuenca-Azuay, lchunirp@ hotmail.com, https://orcid.org/0000-0001-6640-1913

${ }^{2}$ Universidad Católica de Cuenca, Maestría en Desarrollo Local Mención en Ordenamiento Territorial, Cuenca-Azuay, daniele.covri@ucacue.edu.ec, https://orcid.org/0000-0002-2495-0399

${ }^{3}$ Universidad Católica de Cuenca, Maestría en Desarrollo Local Mención en Ordenamiento Territorial, Cuenca-Azuay, ycastilloo@ucacue.edu.ec, https://orcid.org/0000-0002-7710-5199
} 
environmental variables, employment, education, infrastructure, soils, among others; this will allow us to determine effects and interactions that could be generated within the locality; it culminates by posing a theoretical model itself that will serve to carry out the evaluation of possible impacts that could occur in certain variables and based on this model an action plan can be established to mitigate or correct the effects. Conclusions: The Chumblín parish, being an area of a direct influence of the Loma Larga Mining Project, has benefited from projects focused on different axes and family incidence, this through agreements signed annually with the local government, however, it is considered compatible with the good living that is the base paradigm of the development model of the country and that implies obtaining the resources that satisfy the needs of its inhabitants without compromising the natural resources.

Keywords: Incidence, mining, social development, Loma Larga mining project, impact.

\section{Resumen.}

Introducción: La minería no es una actividad nueva, pues desde épocas prehispánicas se tiene indicios de una minería artesanal en donde no se consideraba la importancia de conservar el medio ambiente, conforme pasa el tiempo, esta ha mejorado hasta llegar a procesos de minería a gran escala en donde, para la extracción del mineral se tiene que cumplir parámetros muy exigentes que garanticen el buen vivir de los seres humanos; es decir, se tiene que practicar una minería sustentable y sostenible. Hoy en día la actividad minera es un aspecto relevante dentro del país, puesto que se lo considera como emblemático y estratégico dentro de economía y desarrollo no solo local sino también a nivel nacional. Objetivo: La presente investigación determinará cómo el Proyecto minero Loma Larga incide en el desarrollo social de la parroquia Chumblín. La presente investigación determinará cómo el Proyecto minero Loma Larga incide en el desarrollo social de la parroquia Chumblín. Metodología y Resultados: La investigación se basa fundamentalmente en un análisis documental; en primera instancia del Proyecto minero Loma Larga bajo la concesión de la empresa INV Minerales; luego se hace un estudio de los principales indicadores la parroquia Chumblín, población, nivel de instrucción, índice de envejecimiento, juventud, dependencia, NBI, PET, PEA, PEI, actividad económica, y acceso a servicios básicos mismos que permiten establecer un diagnostico actual del área de estudio para continuar con una investigación de las variables sociales y ambientales, empleo, educación, infraestructura, suelos, entre otras; esto nos permitirá determinar efectos e interacciones que pudieren generarse dentro de la localidad; se culmina planteando un modelo teórico mismo que servirá para realizar la evaluación de posibles impactos que pudieran presentarse en ciertas variables y en base a este modelo se podrá establecer un plan de acción para mitigar o corregir los efectos. Conclusiones: La parroquia Chumblín al ser área de influencia directa del Proyecto Minero Loma Larga ha sido beneficiado de proyectos enfocados en distintos ejes y de incidencia familiar, esto mediante convenios firmados anualmente con el gobierno local sin embargo, se considera compatibles con el buen vivir que es el paradigma base del modelo de desarrollo del país y que implica obtener los recursos que satisfagan las necesidades de sus habitantes sin comprometer los recursos naturales. 
Palabras claves: Incidencia, minería, desarrollo social, Proyecto Loma Larga, impacto.

\section{Introducción.}

En Ecuador, la actividad minera de carácter artesanal comienza desde épocas prehispánicas, hace más de 400 años, pero es en el Gobierno de Rafael Correa en el año 2010 cuando se impulsa con mayor intensidad como parte de un nuevo modelo basado en la explotación de recursos naturales no renovables específicamente petróleo y minerales metálicos. La extracción industrial a gran escala de minerales como el oro, cobre entre otros se fundamenta en suplir la explotación de petróleo en su papel de principal generador de recursos para el país. Esto ha conllevado a que se presenten algunos conflictos socio-ambientales basados en:

- Uso y explotación de la naturaleza;

- Necesidad del Estado de generar excedentes económicas para satisfacer la demanda de la población y,

- Atención de los derechos económicos, sociales y políticos de las comunidades locales involucradas en los proyectos mineros.

Según el Plan Nacional de Desarrollo Minero 2020-2030, el $1.6 \%$ de aporte al Producto Interno Bruto del Ecuador corresponde al sector minero, porcentaje que se encuentra por debajo del nivel de varios países de la región, sin embargo la balanza comercial del sector minero, en el periodo 2010 - 2019, muestra un superavit. Los principales destinos de exportación del sector minero fueron Emiratos Árabes Unidos, India, Estados Unidos de América, Turquía y Suiza.

Con la presente investigación, se dispondrá un instrumento de análisis en donde se mostrará de manera clara y concisa a la sociedad las principales variables de los posibles impactos tanto positivos como negativos que genera la presencia del Proyecto Minero Loma Larga para lo cual se analizaran diferentes variables que involucran el desarrollo de este proyecto.

Al final, se presenta un modelo teórico de evaluación de impactos, el cual tiene el objetivo describir y explicar las fases de la evaluación de una forma clara. En la actualidad el proyecto Loma Larga se encuentra en la fase de exploración avanzada y comenzará su producción a partir del año 2022, razón por la que es imposible realizar una evaluación global de impactos dentro del presente estudio.

Loma Larga es un proyecto minero ubicado en la provincia del Azuay, a $30 \mathrm{~km}$ al suroeste de la ciudad de Cuenca, al sur del Ecuador en la Cordillera Occidental de los Andes. Posee el título de tres concesiones mineras denominada Cerro Casco, Río Falso y Cristal en un área de 8.000 hectáreas aproximadamente. 


\begin{tabular}{cc}
\hline CONCESIÓN & AREA (ha) \\
\hline Cerro Casco & 2552 \\
\hline Rio Falso & 3168 \\
\hline Cristal & 2240 \\
\hline TOTAL & $\mathbf{7 9 6 0}$
\end{tabular}

Tabla 1. Área concesionada por INV Minerales. Fuente: NI 43-101 FEASIBILITY STUDY TECHNICAL REPORT.

Se puede acceder a través de $40 \mathrm{~km}$ de carretera pavimentada desde la ciudad de Cuenca hasta la parroquia San Gerardo y $18 \mathrm{~km}$ de camino empedrado. Loma Larga se encuentra ubicada a una altitud de 3.500 a $3.900 \mathrm{~m}$ sobre el nivel del mar.

La primera exploración sistemática conocida en el área de Loma Larga fue llevada a cabo por las Naciones Unidas a finales de los años setenta para definir las anomalías de los metales básicos. En 1991 fue adquirida por COGEMA (ahora AREVA) que completó una perforación de diez hoyos por un total de $1.869 \mathrm{~m}$ de perforación de diamante en vetas y objetivos diseminados. La mineralización de oro, plata y cobre fue interceptada y, en 1993, Newmont Mining y TVX Gold se convirtieron en socios de la joint venture de AREVA. Entre 1994 y 1996 se ejecutaron otros 7.581 m de perforación de diamante en 82 pozos poco profundos. En 1999 IAMGOLD Corporation (“IAMGOLD”) adquirió la propiedad de AREVA y es a partir del año 2003 que comenzó la exploración en las concesiones de Cerro Casco y Rio Falso. En el 2004 se descubre el yacimiento que comprendía una ley de 9,5 g/t, 46,7 g/t de plata y 0,40\% de cobre contenidos en 101,4 m. Se perforó un total de 65.117 m entre 2003 y 2008, en este mismo año el Gobierno Central del Ecuador decretó una suspensión de toda actividad de exploración y de explotación, pero a partir del 12 de enero de 2009, el Gobierno del Ecuador aprueba una nueva ley minera con el objeto de impulsar esta actividad. En el año 2012 la empresa INV Metals era ya propietaria unica del 100\% de la propiedad Loma Larga. En el 2013 la Compañía concluyó su programa de exploración inicial. El programa comprendía 12 pozos de perforación de diamante por un total de $3.684,7 \mathrm{~m}$, incluyendo pozos de pruebas de objetivos para ampliar el yacimiento, pozos más profundos para probar las lentes apiladas y pozos para obtener muestras para pruebas metalúrgicas. La Compañía también concluyó un programa de trabajo de pruebas metalúrgicas. Los resultados de los cuales comprenden un circuito de flotación de dos etapas; en julio de este mismo año, el gobierno ecuatoriano enmendó sus leyes mineras y creó una nueva categoría de minería de mediana escala. La información publicada hasta la fecha indica que la producción subterránea clasificada en esta categoría estaría limitada a 1.000 toneladas por día y estaría sujeta a impuestos sobre la renta para las sociedades, a un $4 \%$ de oro y derechos de subproducto, impuestos al valor agregado, $3 \%$ de participación de los empleados en las utilidades, $12 \%$ de participación de los beneficios estatales en proyectos de desarrollo social, y un ajuste soberano. Como un proyecto de minería de mediana escala, el proyecto no estaría sujeto a los mismos requisitos que la categoría de minería a gran escala, que incluye la negociación de un acuerdo de explotación, un mínimo de 5-8\% de oro y derechos de subproductos, regalías anticipadas e impuestos especiales. 
Según el portal institucional en el 2018 la Compañía finalizó el estudio de prefactibilidad en Loma Larga en donde uno de los resultados obtenidos es que se tendrá tasa de producción de aproximadamente 3.000 toneladas por día y de 206.000 onzas de oro anualmente. Este estudio de prefactibilidad estima además una tasa de interna de retorno TIR del $24.7 \%$ después de impuestos con una recuperación de 2.6 años, el estudio de factibilidad confirma entonces la viabilidad del proyecto y una sólida rentabilidad financiera del proyecto de oro-plata-cobre. Durante 12 años de vida útil proyectada, se prevé la extracción de 2.6 millones de onzas de oro equivalente, con un promedio de 227,000 onzas de oro equivalente extraídas por año y un promedio de 294,000 onzas de oro equivalente extraídas durante los primeros cuatro años completos (INV Metals, s.f.)

La zona en donde se realizará la explotación del mineral es un páramo de alta biodiversidad que alberga más de treinta lagunas en un sistema hídrico del que surgen los tres ríos principales de los cuatro que tiene Cuenca y sus once micro - cuencas. Para los indígenas Kañari, consideran este espacio como un lugar sagrado.

En el año 2012 la empresa canadiense IamGold vendió su proyecto Quimsacocha. La razón para la venta del proyecto se asume estuvieron relacionadas con el constante conflicto que tenían con los moradores de la zona afectada. La empresa no logró el consentimiento de los pueblos que se verían afectados por este proyecto ante la posibilidad de contaminación de las fuentes de agua dulce, daño que se considera como irreversible. Debido a esto, en octubre de 2011 se realizó una consulta popular en las comunidades de Victoria del Portete y Tarqui y el 92\% de población voto en contra de la Minería en Quimsacocha (Vega, 2018).

Uno de los objetivos estratégicos del Plan Nacional de Desarrollo del Sector Minero es el de:

"desarrollar procesos de planificación especial en áreas de influencia de proyectos de trascendencia nacional y zonas económicas de desarrollo especial, con consulta previa, libre e informada, veeduría ciudadana y control social",

en la actualidad el Ecuador encuentra en la minería responsable una gran oportunidad para su crecimiento y desarrollo, sostenida en una actividad técnica y profesional señida a exigentes niveles de cuidado ambiental, beneficio social y seguridad por lo que el proyecto minero Loma Larga es considerado un referente dentro del sector Minero Industrial debido al avance tecnológico y científico que propone, impulsado por expertos ecuatorianos de amplia trayectoria, quienes desde hace 20 años trabajan bajo la premisa de evolucionar hacia un nuevo modelo de esta actividad productiva en el Ecuador. INV Metals como empresa operadora del Proyecto Loma Larga desde hace muchos años está a tono con los nuevos paradigmas ambientales. Por ello, ha logrado desarrollar modelos que permitirán optimizar el uso de los recursos en las siguientes etapas de la actividad minera (INV Metals, 2020). 
Según información dotada por la empresa, el diseño de la mina del Proyecto Loma Larga es subterráneo, esto hace pensar que la superficie y condiciones del suelo no van a ser alterados de esta manera se evita daños al entorno de toda la zona, se aclara además que gracias a la investigación y las nuevas tecnologías se dispondrá de un modelo mejorado de la Planta de Concentrados, pues en el sitio no se refinará ningún tipo de material, asegurando que el recurso hídrico y mineral no tendrá contacto con elementos químicos. El recurso hídrico para las operaciones provendrá en mayor parte del agua lluvia, se establece además que, cumplida la vida útil de la mina subterránea, la planta de concentrados será rehabilitada.

La minería subterránea, a diferencia de la explotación a cielo abierto, requiere de mayor especialización, debido a la variedad de operaciones unitarias que son necesarias, como perforación, tronadura, ventilación, manejo de materiales, geomecánica, entre otros procedimientos. Existen, además, varias técnicas para explotar un yacimiento de manera subterránea que dependerá del tipo de material o rocas que se encuentre formadas en el yacimiento, por lo que es necesario una planificación con mayor tecnicidad. Las condiciones de trabajo dependerán tanto del mineral que se encuentre, como de la profundidad y uso de maquinaria (Reforma Minera, 2009).

Pese a la existencia de estas técnicas de extracción, la población muestra preocupación sobre la contaminación y destrucción ambiental que ocasionaría en la geografía de la zona pese a disponer de diversos estudios de impacto ambiental exigidos por los entes gubernamentales como el Ministerio del Ambiente, Ministerio de Minas, SENAGUA, Municipios, Juntas Parroquiales y ETAPA.

La minería causa un gran impacto por ser un eje central de desarrollo y sustento de las comunidades y familias donde este está implementado. Así tiene aspectos positivos como: desarrollo de la comunidad; fomenta la unión, es una fuente externa de economía, y en sentido opuesto: no cumple con las leyes que la rigen, se dan fatales accidentes por mínima seguridad, se constituye como un eje de problemas económicos y sociales. Además, la minería, por ser una fuente de desarrollo, también es una fuente de ingresos y egresos, ya que con el dinero que se obtenga de los minerales, se tendrá que cubrir una serie de gastos como: remuneraciones directas, energía, contratistas y consultores, servicios generales y de mantención, combustibles y lubricantes, insumos de planta, las maquinarias que ayudan a realizar dicho trabajo (Gudynas ,2009).

La ley de minería del Ecuador del año 2011, obliga a los concesionarios mineros a realizar estudios de impacto ambiental y planes de manejo, señalan el contenido de los planes de manejo, exige medidas de tratamiento de aguas, de reforestación, conservación de flora y fauna, protección de los ecosistemas, obliga a tener un buen manejo en cuanto a los desechos y acumulación de los residuos provenientes de las actividades mineras. (Ley de Minería, 2011).

Algunas malas practicas en las actividades mineras han producido impactos ambientales, 
al microclima, modificación de la geografía de la zona, erosión, alteración de ecosistemas, impactos socioeconómicos, entre otros. Por ello, que la minería presenta daños que han sido evidentes, y por esta razón existen los levantamientos de grupos de población que viven en zonas de influencia directa de los proyectos mineros, y que han sido los primeros actores en rechazar la actividad minera en zonas cercanas a ellos generando muchas tensiones (Chapa \& López 2016).

El Ingeniero Geólogo (Edgar Pillajo, 2011) menciona que: "al no tomar medidas preventivas, la actividad minera puede afectar al ambiente físico en los componentes agua, suelo, aire, clima". Si no se considera evaluaciones de impacto ambiental y planes de manejo ambiental, si no existieran nuevos métodos tecnológicos para disminuir impactos ambientales, si no existen garantías de fiel cumplimiento de las empresas mineras mediante de un fidecomiso, si las comunidades cercanas al proyecto no se organizan para conformar las veedurías ciudadanas, los impactos ambientales serán mayores (Pillajo, 2011:194). Ante esto, se da la necesidad de realizar acuerdos entre Estado, comunidades y empresas privadas para un óptimo desarrollo local y minero. Según, (Pillajo, 2011:316). Una minería que se desarrolle en base a lo que dicta la Constitución y las Leyes ecuatorianas actuales, en donde hasta la naturaleza es sujeta de derechos mineros, sin duda alguna aportará al Buen Vivir no solo de los ecuatorianos que viven en la localidad sino de todos a nivel nacional. Gustavo Morejón especialista en temas ambientales, ex subsecretario regional de Medio Ambiente en el Azuay, mencionó a la prensa: "casi todo lo que gira a nuestro alrededor y de lo cual nos servimos a diario, como la luz eléctrica o las vías, está vinculado al quehacer minero, oponerse ciegamente significaría tener que aislarse en una caverna y retroceder en la historia; hay que hacer responsablemente las cosas". (Diario El Mercurio, 09/22/2011)

En la localidad Chumblín se encuentra hoy en día involucrada directamente con el tema, gracias a la presencia del proyecto minero Loma Larga; Chumblín está ubicada dentro del área de influencia directa del Proyecto Loma Larga dentro del cantón San Fernando, de la Provincia del Azuay, en la Región Interandina de la República del Ecuador.

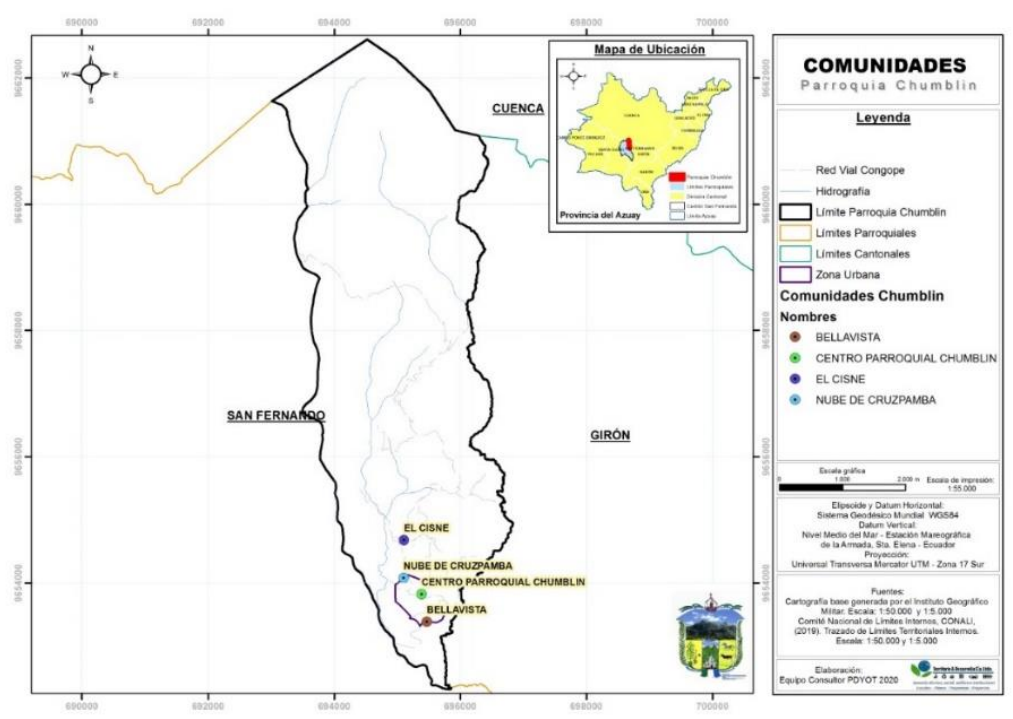

Figura 1. Ubicación general de la parroquia Chumblín Fuente: PDOT Chumblín 2020. 


\begin{tabular}{|c|c|}
\hline Población & 749 habitantes \\
\hline Superficie & $\begin{array}{l}\text { Chumblín se localiza al suroeste de la ciudad de Cuenca, provincia del Azuay, cuenta } \\
\text { con una superficie de } 23 \text { kilómetros cuadrados. }\end{array}$ \\
\hline Altitud & 2500 a $4000 \mathrm{msnm}$ \\
\hline Clima & Frío \\
\hline Temperatura & $3^{\circ} \mathrm{C}$ a $16^{\circ} \mathrm{C}$ \\
\hline Topografía & $\begin{array}{l}\text { La topografía es moderada irregular (quebrada), la mayor parte de los terrenos se } \\
\text { caracterizan por ser pedregosos, con pendientes de hasta el } 20 \% \text {, dificultando en gran } \\
\text { parte las actividades agrícolas; sin embargo, son terrenos fértiles y aptos para el cultivo } \\
\text { de maíz, papas, frejol, habas y pastos }\end{array}$ \\
\hline Vialidad & $\begin{array}{l}\text { La vía de acceso desde Cuenca es totalmente asfaltada, la distancia desde esta urbe a } \\
\text { la cabecera parroquial es de } 75 \text { kilómetros y el periodo de desplazamiento en vehículo } \\
\text { es de } 1 \text { hora } 30 \text { minutos. }\end{array}$ \\
\hline Comunidades & $\begin{array}{l}\text { Centro Parroquial, Recinto Bellavista, Recinto la Nube de Cruzpamba y Recinto el } \\
\text { Cisne. }\end{array}$ \\
\hline
\end{tabular}

Tabla 2. Datos generales de la parroquia Chumblín

Fuente: PDOT Chumblín 2020.

\section{Principales Indicadores}

La pirámide poblacional de un sector muestra la estructura y composición de la población según grupos de edad y sexo. En el caso de la parroquia Chumblín, la población guarda una estructura de pirámide poblacional particular, observándose un comportamiento particular en tanto que existe una leve disminución en la tasa de natalidad de la población, factor que puede ser motivado por un cambio cultural hacia una tendencia a la disminución del número de hijos por familia, así como al retraso en la edad para concebir. Se observa también que existe una disminución de la población a partir de las edades de 14 años; con una tendencia más pronunciada en la población masculina, tendencia evidente hasta los 40 años; factor que puede explicarse por la salida de la población por razones de estudio y/o trabajo hacia la ciudad por las mayores oportunidades laborales y educativas. Esto es mayor en hombres que en mujeres. La población adulta mayor pierde representatividad a partir de los 80 años.

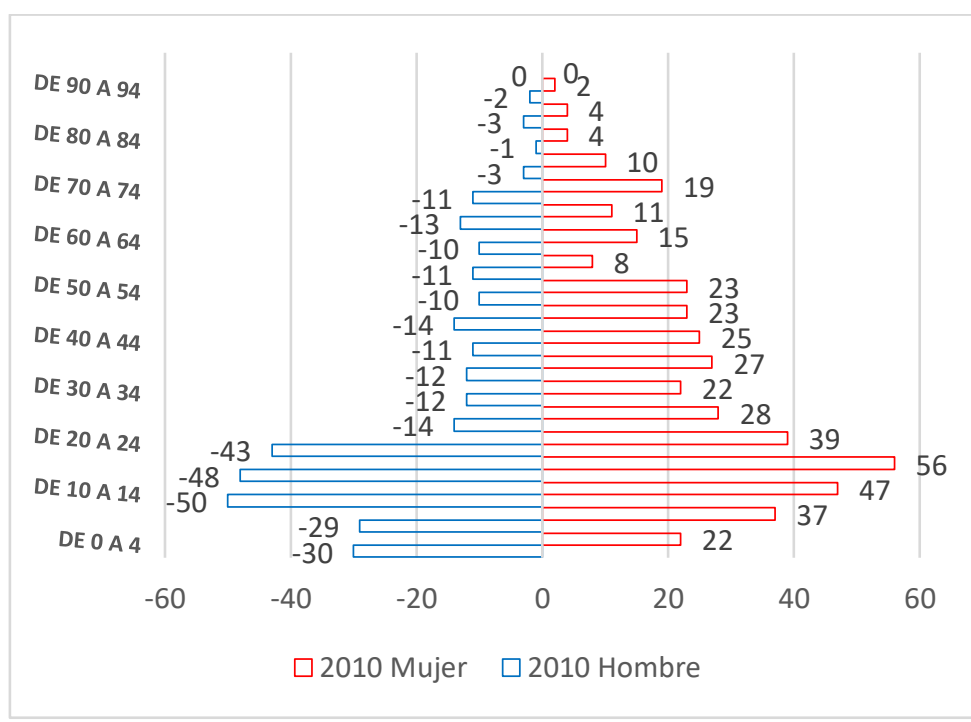

Figura 2. Pirámide poblacional Chumblín

Fuente: PDOT 2020. 
En la parroquia Chumblín el $72 \%$ de la población solo ha alcanzado un nivel de educación primaria o básica, solo el 5,54\% alcanza la educación media y apenas un 4,52\% ha tenido acceso a la educación superior.

La tasa de analfabetismo en la parroquia Chumblín es del $7.5 \%$ porcentaje que indica que 7 de cada 100 personas mayores de 15 años no saben leer y escribir.

\begin{tabular}{lc}
\hline \multicolumn{1}{c}{ NIVEL DE INSTRUCCIÓN } & CHUMBLÍN \\
Ninguno & $4,81 \%$ \\
Centro de Alfabetización/(EBA) & $0,73 \%$ \\
Pre escolar & $0,00 \%$ \\
Primario & $\mathbf{5 8 , 6 0 \%}$ \\
Secundario & $11,22 \%$ \\
Educación Básica & $\mathbf{1 3 , 9 9 \%}$ \\
Educación Media & $5,54 \%$ \\
Ciclo Post bachillerato & $0,58 \%$ \\
Superior & $4,52 \%$ \\
Postgrado & $0,00 \%$ \\
TOTAL & $\mathbf{1 0 0 , 0 0 \%}$ \\
\hline
\end{tabular}

Tabla 3. Nivel de Instrucción

Fuente: Censo de Población y Vivienda 2010.

El índice de envejecimiento mide la proporción de la población adulta mayor con respecto a la población menor de 15 años. Este indicador muestra que por cada 100 niños y adolescentes menores de 15 años existen 12 adultos mayores, situación que se corresponde con la disminución de los niveles de natalidad de la población. Por su parte el índice de juventud es del $30 \%$, que implica que, de cada 100 habitantes, 30 son jóvenes entre 15 y 29 años. La parroquia Chumblín tiene una tasa de dependencia de 66.08\%; considerándose como dependiente la población adulta mayor y la menor de 15 años. La tasa de dependencia de $66 \%$ indica que por cada 100 personas en edad productiva existen 66 que dependen de estas, es decir, la población que necesita de la fuerza generadora de recursos está cercana a una relación de uno a uno. Esto implica que existe una fuerte carga para la población no dependiente, que sumado a los niveles de juventud bajos y al índice de envejecimiento, necesita atención este indicador. La población que se encuentra en edad productiva al tener un número mayor de dependientes limitaría su capacidad de ahorro e inversión de la población.

\begin{tabular}{lclclc}
\hline $\begin{array}{l}\text { Población de 65 } \\
\text { años y más }\end{array}$ & 83 & $\begin{array}{l}\text { Población de } 15 \text { a } \\
29 \text { años }\end{array}$ & 228 & $\begin{array}{l}\text { Población } \\
\text { dependiente }\end{array}$ & 298 \\
\hline $\begin{array}{l}\text { Población menor } \\
\text { de } 15 \text { años }\end{array}$ & 666 & $\begin{array}{l}\text { Población } \\
\text { total }\end{array}$ & 749 & $\begin{array}{l}\text { Población no } \\
\text { dependiente }\end{array}$ & 451 \\
\hline $\begin{array}{l}\text { Índice de } \\
\text { envejecimiento }\end{array}$ & $\begin{array}{c}\mathbf{1 2 . 4 6} \\
\text { Índice de }\end{array}$ & $\begin{array}{l}\text { juventud } \\
\text { jundice de }\end{array}$ & $\mathbf{3 0 . 4 4 \%}$ & $\begin{array}{l}\text { Independencia } \\
\text { depend }\end{array}$ & $\mathbf{\%}$ \\
\hline
\end{tabular}

Tabla 4. Índice de envejecimiento, juventud y dependencia

Fuente: Censo de población y vivienda 2010.

La pobreza por necesidades básicas insatisfechas es un indicador directo de medición de la pobreza; es decir, considera las carencias que tiene la población, más allá de su nivel de ingresos. Para la medición de la pobreza por necesidades básicas insatisfechas, se considera 5 dimensiones, las mismas que son: hacinamiento, acceso a vivienda, acceso a 
educación, acceso a Servicios Básicos y, Capacidad Económica. Se considera que la falta de una de estas dimensiones se asume al hogar como pobre. En la parroquia Chumblín la tasa de pobreza por necesidades básicas insatisfechas es del 52,74\% esto implica que más de la mitad de la población vive en hogares que no cumplen con las 5 dimensiones de las necesidades básicas. En lo que se refiere a las comunidades Nube de Cruzpamba y Bellavista, los niveles de pobreza son similares, sin embargo, en la comunidad El Cisne, la tasa de pobreza es superior en casi un $30 \%$ a las demás comunidades, con la particularidad de que se encuentra en el área más dispersa.

\begin{tabular}{cc}
\hline & Tasa de pobreza \\
\hline PARROQUIA CHUMBLín & $\mathbf{5 2 . 7 4 \%}$ \\
Nube de Cruzpamba & $36,52 \%$ \\
Bellavista & $38,78 \%$ \\
El Cisne & $67,15 \%$ \\
\hline
\end{tabular}

Tabla 5. Necesidades Básicas Insatisfechas Chumblín

Fuente: Censo de Población y Vivienda 2010.

De la población total de la parroquia 631 personas ( $84 \%$ del total de la población) están en edad de trabajar (PET) de los cuales 268 son hombres y 363 mujeres representando el $36 \%$ y $48 \%$ respectivamente del total de la población, se determina además que hay una apreciativa oferta laboral de mujeres dentro de la parroquia, el PET es un indicador demográfico que refleja la oferta de trabajo, incluye a las personas de 10 años y más, tanto a las activas cuanto a las inactivas (estudiantes, jubilados y pensionistas, quienes se dedican sólo a quehaceres domésticos). En tanto la Población Económicamente Activa representa el $43.66 \%$ de la población total de los cuales 171 son hombre y 156 son mujeres. La PEA está conformada por las personas de 10 años y más que trabajaron al menos 1 hora, y aquellas personas que no tenían empleo, pero estaban disponibles para trabajar y buscaban empleo (desocupados).

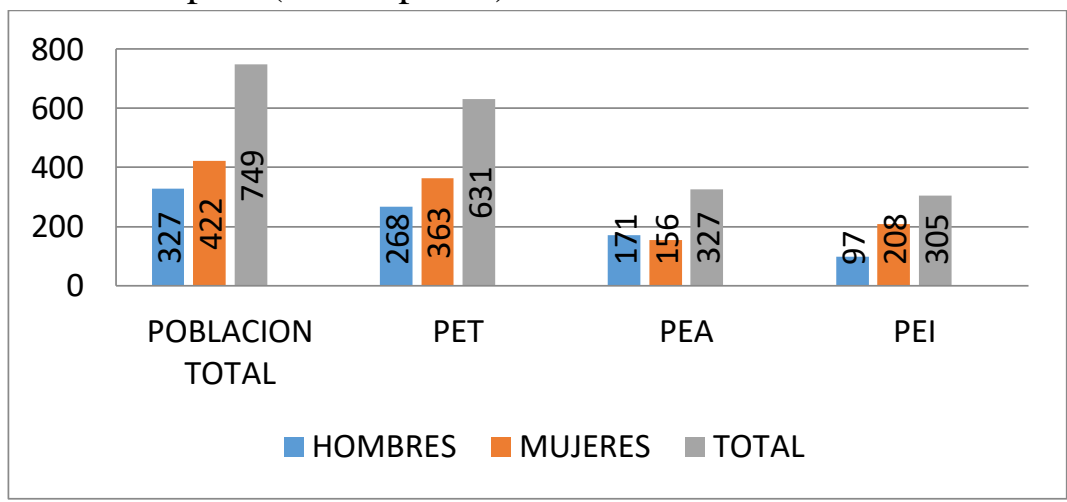

Figura 3. Indicadores PET, PEA y PEI.

Fuente: Censo de población y vivienda 2010.

La actividad económica, nos permite clasificar al establecimiento donde trabaja o trabajó la persona dentro de un sector de la economía, según la clase de bienes o servicios que produce la PEA de la parroquia Chumblín, el 58.10\% se dedica a actividades del sector primario esto es actividades de agricultura, ganadería, silvicultura y pesca de lo cual 86 son casos de hombre y 104 son mujeres determinándose una vez más de forma apreciativa que existe más oferta laboral de mujeres dentro de la parroquia. 


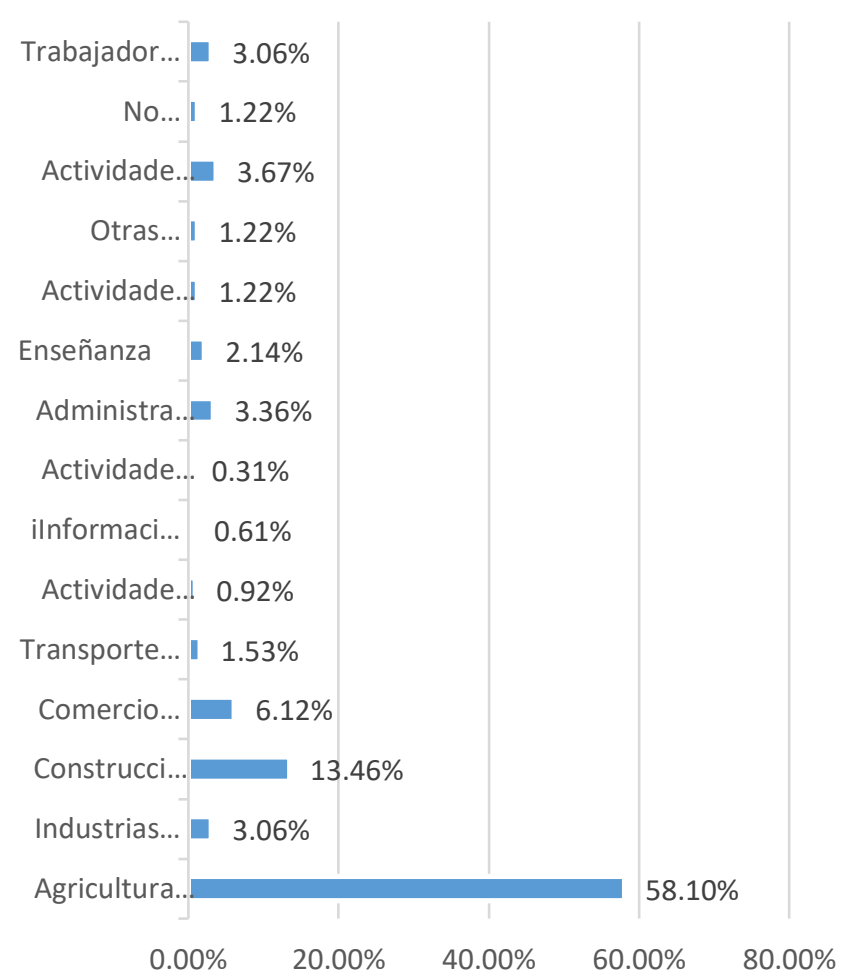

Figura 4. PEA según grupo de actividad

Fuente: Censo de Población y vivienda 2010.

El $92 \%$ de las viviendas de la parroquia Chumblín, tienen acceso a agua potable que proviene de red pública, situación beneficiosa para la población, sin embargo, aún existe en menor porcentaje las familias que se abastecen de pozo y de canal acequia: $2 \%$ y $6 \%$ respectivamente.

La mayoría de las viviendas presentan como forma de evacuación de excretas el alcantarillado con un $63 \%$ del total de viviendas, es decir más de la mitad de las viviendas ocupadas con personas presentes disponen de alcantarillado, aspecto positivo desde el punto de vista social y medio ambiental.

En la parroquia Chumblín la predominancia en la eliminación y disponibilidad del servicio de desechos sólidos corresponde a por medio de carro recolector, con un $80 \%$, de las viviendas, seguido de 7,5\% de viviendas que la queman, $7 \%$ la arrojan a quebradas o ríos, un $5 \%$ la entierran.

En la parroquia Chumblín, el 98,5\% de las viviendas tienen acceso al servicio de energía eléctrica procedente de red pública, cubriendo casi en su totalidad a las 210 viviendas ocupadas y, apenas el 1,5\% de la población no dispone de este servicio.

El análisis de las dimensiones sociodemográficas y sanitarias permite disponer de una visión importante sobre la estructura poblacional. Se evidencia una población con tendencia al envejecimiento por la disminución de natalidad generando a su vez mayores niveles de dependencia. Dentro de las dimisiones sanitarias se evidencia que las 
dimensiones incidentes para la tasa de pobreza por NBI están vinculadas con el agua para consumo humano.

\section{Metodologia.}

El presente trabajo de investigacion es de tipo cualitativo, pues pretende analizar algunas variables en donde se intuye posible incidencia como efecto del desarrollo de la actividad minera dentro de la parroquia; un estudio descriptivo pues se hace una descripcion de la mineria en la parroquia Chumblín; un estudio transversal observacional, pues la recolección de datos se la realiza en un solo momento, a través del análisis de la información disponible sobre el Proyecto Loma Larga.

En base al tipo de investigación planteada, se cree conveniente utilizar el método Inductivo-Deductivo, este metodo permite estudiar la problemática planteada desde las partes hacia un todo, con el análisis se conocera los elementos y características del proceso económico social cuyos impactos se pretende conocer para lograr, a través de la síntesis conclusiones y resultados generales de la investigación.

El método inductivo intenta ordenar la observación tratando de extraer conclusiones de carácter universal desde la acumulación de datos particulares.

La modalidad de la investigación es documental, es decir, está basada en la búsqueda bibliográfica y de documentos tanto de conceptos, criterios y teorías; así también, como de información estadística sobre el tema a tratar.

\section{Resultados}

La actividad predominante de la zona de influencia directa del proyecto es la ganadería y la agricultura. La producción de leche es la que mayores réditos brinda a las familias. Años atrás el principal tipo de agricultura era de autoconsumo.

Hoy en día, la realidad social ha cambiado debido a variaciones en varios contextos sociales y económicas como resultado de la influencia de la actividad minera en las prácticas sociales y circunstanciales de Chumblín.

Al realizar el análisis de las variables sociales y ambientales, mismas que han sido agrupadas en tópicos como, por ejemplo: empleo, educación, infraestructura, hidrología, suelos, entre otras; esto nos permitirá determinar efectos e interacciones que pudieren generarse entre ellas.

Empleo. - El sector minero contribuye a la dinamización de la economía local ya que la generación de plazas de trabajo tanto de forma directa como indirecta, mismo que implica el ámbito productivo y comercial de la parroquia.

Según las expectativas el Proyecto Loma Larga durante el período de construcción de la mina que durará de 18 a 24 meses, otorgará empleo directo estimado de 875 personas; cuando la mina esté en operación, un estimado de 450 empleos directos permanentes; además la creación de puestos de trabajo indirectos con iniciativas de contratación local 
y oportunidades de capacitación; los salarios, la seguridad social y los beneficios de pensiones se estiman en \$15 millones anuales, para un total de \$186 millones a lo largo de la vida útil de la mina; el monto de la participación de los trabajadores en las utilidades (3\%) se estima en $\$ 34$ millones. $^{4}$

El empleo indirecto generado por la actividad minera se lo conoce como "efectos multiplicadores". El efecto multiplicador varía según la cantidad y tipo de contratos con proveedores de bienes y servicios. Por lo general en el sector minero la cantidad de empleo indirecto es mayor al empleo directo (Zeballos y Garry 2010).

La minería es la "madre de las industrias" se vincula con el importante efecto multiplicador que genera encadenamientos productivos, empleo, flujo de ingresos a partir de las inversiones y compra de insumos nacionales, generación de divisas, innovación técnica y tecnológica, desarrollo de infraestructura, recursos para inversión en salud, educación, desarrollo local, capacitación y entrenamiento para gestión del talento humano para una minería bien hecha (Ministerio de Recursos Naturales no Renovables 2015, 8) La contratación externa de bienes y servicios estimula el emprendimiento y el desarrollo de nuevos negocios en las comunidades circundantes, además porque existe una mayor inyección monetaria en la localidad por parte de los empleados y contratistas debido a que sus sueldos son mayores que el nivel general promedio de los salarios locales, los mismos que gastan en la economía local, en consecuencia con el transcurso del tiempo el número de empleos indirectos y los "efectos multiplicadores" tiende a aumentar.

En el Ecuador la generación de empleos en el sector minero proviene de la extracción de oro o de materiales de construcción, es así que en año 2010, de los 6.888 empleos creados, 4.724 corresponden a minas de oro y 1.574 a yacimientos de materiales de construcción (Ordeñana y Dominguez 2015).

Según el Plan Nacional de Desarrollo para el 2021 se espera que la minería aporte el 4\% al PIB; además establece un fuerte fortalecimiento de las economías, creación de empresas y encadenamientos productivos destinados a satisfacer las necesidades de la industria minera. Estudios internacionales estiman que por cada empleo directo en minería se generan de 3 a 5 trabajos indirectos.

Educación. - Se establece que la construcción de una mina del Proyecto Minero son las que generan más empleo; sin embargo, esta fase de construcción suele ser muy corta aproximadamente entre 18 a 24 meses a comparación de la fase de operación que tiene un periodo de duración entre 120 a 240 meses, en esta etapa se requiere mano de obra calificada, con niveles de instrucción y escolaridad que la mayoría de las personas de las comunidades no poseen.

En referencia a las plazas que ofrecen los proveedores de la industria minera también requieren técnicos especialistas, quienes deben cumplir con regulaciones, certificaciones

\footnotetext{
${ }^{4}$ Según reporta la página web INV Metals:

https://sp.invmetals.com/wpcontent/uploads/2020/04/PR_March31_2020_FS_RESULTS_FINA L_ES-MSAM2.pdf
} 
y normas de operación productiva, excluyendo de esta forma de cierta manera a la mano de obra local.

Por otro lado, como lo establecen Bustamante y Lara, "la actividad minera se caracteriza por requerir altos montos de inversión, sin embargo, el pago de la mano de obra representa una fracción pequeña del valor agregado de la producción, por lo tanto, el impacto directo en el empleo es más reducido que lo que se podría suponer" (Bustamante y Lara 2010, 41).

Por ende, si bien el proyecto minero Loma Larga genera empleo para los habitantes de la zona, también es cierto que será por un periodo corto, ya que la mano de obra que requiere el proyecto deberá ser más calificada y contar con niveles de escolaridad y preparación técnica avanzada, requisito que no lo pueden cumplir los pobladores, cabe señalar que representantes del proyecto minero aseguran dotar de capacitaciones a las personas que fueren contratadas.

Infraestructura. - La industria minera requiere de cierta infraestructura para su desenvolvimiento, de ahí, que las empresas que trabajan dentro de esta actividad impulsen la construcción de carreteras y servicios de comunicación, beneficiando el desarrollo de otras actividades económicas. En la parroquia Chumblín pese que aún el proyecto no se encuentra en el proceso de producción la empresa mediante firma de convenios de cooperación con el gobierno local se ha invertido en infraestructura a través de mejoramiento de vías, tecnificación de riego, mejoramiento del Complejo Deportivo, Cementerio Parroquial, Mercado Parroquial, Parque Central, Casas Comunales de los recintos; la anteriormente Empresa denominada Ecuador Estratégico misma que se dedicaba a ejecutar proyectos estratégicos donde está emplazado un proyecto minero es así que por el año 2014 se emprende la construcción de la planta de tratamiento de agua potable y alcantarillado de la parroquia Chumblín mismo que cubre con la dotación al 90 $\%$ de la población de agua potable, sin embargo hasta la actualidad pese a que la obra está concluida esta no es utilizada por cuestiones legales que cumplir.

Migración y revalorización de la tierra.- Una de las consecuencias del establecimiento de las operaciones mineras en una población, son los flujos migratorios, muchas personas se trasladan a las localidades donde se encuentran las minas para trabajar en las diferentes actividades artesanales, agrícolas, comerciales o mineras; los flujos demográficos implican usualmente una revalorización de la tierra, es decir, las propiedades aumentan de precio considerablemente, lo cual beneficia a sus dueños, siempre y cuando hayan asegurado legalmente los títulos de propiedad.

A nivel mundial se aprecia que la minería crea enormes problemas sociales y ambientales, además de desplazar a comunidades enteras generando conflictos violentos en comunidades que se oponen a las minas. Esto no se aprecia en la parroquia Chumblín por un lado porque el proyecto aún no se encuentra en la fase de producción y por otro lado al estar la mina a una considerable distancia de los principales centros poblados se prevé que no haya un despojamiento de población. 


\section{Conflictos socio-ambientales}

Las características de la industria minera, su diversidad en cuanto al tamaño de la inversión y de la infraestructura, el tipo de tecnologías y procesos que utiliza; y su relación con la sociedad y el medio ambiente han producido impactos específicos que enfrentan a los actores involucrados en la lucha por remediar problemas como la contaminación de los ecosistemas y los enfrentamientos y tensiones entre las comunidades locales y las empresas.

El desarrollo de la minería en el Ecuador responde a la búsqueda de nuevos ingresos que ayuden a compensar el desgaste de los ingresos petroleros con los provenientes de otra actividad extractivista de la misma naturaleza; así, al igual que la explotación petrolera, la minería extrae recursos no renovables, provocando impactos socioambientales de gran extensión espacial y temporal que reconfiguran y transforman los ecosistemas y las relaciones territoriales.

Eduardo Gudynas, pone de relevancia la existencia de dos tipos de extractivismo: un extractivismo clásico o convencional, en el que el proceso está a cargo de las empresas transnacionales y los Estados se limitan a un papel regulatorio destinado a proteger los derechos de los ecosistemas, de las poblaciones humanas y los intereses económicos nacionales; $y$, un extractivismo progresista o neo extractivismo que está caracterizado por una mayor presencia del Estado y niveles de tributación mayores, esta forma de extractivismo promete a los habitantes mejores condiciones de vida pero, al tener su origen en las relaciones económicas externas del país, afecta de manera drástica las relaciones socioeconómicas locales (Gudynas 2011, 75).

Según Rosie Kuhn, el mayor riesgo ambiental de la mina en Kimsacocha es el drenaje ácido de Minas (DAM) pues al minar en un yacimiento de mineral de sulfuros y se expone al oxígeno, el agua y las bacterias forman ácidos. Este ácido genera otros metales, entre ellos cobre, plomo, zinc, entre otros que podrían ocasionar efectos adversos perjudiciales para el medio ambiente e incluso puede llegar a afectar a la salud humana (Kuhn, 2011). Pese a que la empresa minera con su alto rango de tecnología avanzada y de punta garantiza la explotación en el lugar, se toma en cuenta que ninguna de estas prácticas puede prevenir este tipo de secuelas y peor aún eliminar los efectos que a la larga repercuten en gran medida a la población y a su vegetación. Por ende, se asume lo que menciona Alberto Acosta y William Sacher (Sacher y Acosta 2012, 79) quienes plantean que los posibles impactos más importantes en el ámbito social son:

- El desplazamiento de las poblaciones.

- La pérdida de los derechos colectivos, territoriales y ambientales.

- La transformación de la relación del ser humano con la naturaleza.

- La destrucción de las bases materiales de los pueblos indígenas.

- La implantación de nuevos imaginarios de consumo y modos de vida. 
- La concentración de los poderes, esto redifine la estructura social tanto de las comunidades en donde se desarrolla la actividad minera como de sus alrededores.

- La generación de conflictos al interior de las comunidades.

- Marginación socioeconómica de sectores que no estan preparados o capacitados para el trabajo minero y actividades económicas conexas que éste genera, en especial de mujeres y campesinos.

Por lo tanto, los impactos de cualquier tipo de minería -pequeña o a gran escala- se perciben en las cercanías de las minas, así como también a cientos de kilómetros de distancia. Las minas en las montañas depositan su contaminación en los ríos aledaños, y envenenan a los organismos acuáticos en el recorrido de los ríos hasta llegar al mar. Si las aguas tóxicas son utilizadas para el riego, las plantas captan elementos tóxicos como el arsénico y plomo y los transfiere a las frutas y verduras; en el mar, los metales pesados $\mathrm{y}$ otras sustancias tóxicas son asimilados por mariscos y peces, esto repercute directamente en la economía de pescadores, recolectores de conchas e incluso las camaroneras e intoxican a los consumidores.

Dentro de la parroquia Chumblín, lugar de análisis de acuerdo al diario digital Primicias se establece que:

- El Proyecto Loma Larga presenta un diseño de mina subterráneo. Por lo que se establece que la geografía de la superficie en donde esta emplazada la mina no será alterada y de esta manera se evitaran afectaciones al entorno.

- Realizar minería subterránea permite el manejo de la geografía ambiental de zona ya que en base al desarrollo de la investigación y la disposición de tecnologías avanzadas se construirá un modelo perfeccionado y optimizado de una planta de concentrados de menor tamaño. El Proyecto Minero Loma Larga no refinará ningún tipo de material en la zona. De esta forma se garantiza que el recurso hídrico y mineral no sea contaminado con sustancias químicas utilizadas.

- Para las operaciones dentro de la mina el recurso hídrico se obtendrá en mayor parte, del agua lluvia y el consumo será según las normas y permisos vigentes por los entes pertinentes con un moderno sistema de recirculación y reciclaje.

- Al termino de la vida útil de la mina subterránea del Proyecto Loma Larga, la planta de concentrados será rehabilitada cumpliendo cada uno de los estándares más exigentes de este tipo de industria.

- La inversión total del proyecto minero supera los 500 millones esto conlleva a que gran parte de las familias de los lugares de incidencia directa y sus alrededores sean beneficiadas con empleo y también como proveedores de bienes y servicios requeridos.

- Dentro del país, el proyecto minero Loma Larga es el más investigado en el componente ambiental es así que tiene establecido 18 puntos de monitoreo, cuyo objeto es medir las condiciones del agua lluvia y de esta manera determinar comportamiento hidrológico de la zona. Con esta información se aplican modelos 
que para conservar las características del recurso hídrico en todas las fases de la actividad minera, desarrolladas sin afectación ambiental. Instituciones como ETAPA, iDRHICA, PROMAS, Universidad del Azuay y la Universidad Politécnica Salesiana, también realizan estudios de investigación científica del clima, suelo, agua y vegetación del área concesionada. En la actualidad tareas de rehabilitación en algunos espacios intervenidos se han recuperado el 98\% de sus características naturales, conforme establecen monitoreos técnicos.

- Los programas y proyectos de INV Metals están articulados directamente a los Planes de Desarrollo y Ordenamiento Territorial locales. Disponer de este documento de planificación hace que la participación del Estado y de la Empresa Privada INV Metals fortalezcan los proyectos de la parroquia, recintos y mas actores sociales. Desde el año 2009 aproximadamente se ha construido una relación armónica con toda la parroquia, se ha logrado la firma de convenios interinstitucionales para la ejecución de proyectos de capacitación, salud, recuperación de tradiciones, fomento de talento humano local, producción e impulso a emprendimientos innovadores, etc. Fortaleciendo la estructura y desarrollo de mas de 30 organizaciones locales.

\begin{tabular}{cc}
\hline AÑO & MONTO INVERSIÓN \\
\hline $2009-2010$ & 82300 \\
\hline $2011-2012$ & 90000 \\
\hline $2012-2013$ & 90000 \\
\hline 2016 & 20000 \\
\hline $2017-2018$ & 55000 \\
\hline $2018-2019$ & 60000 \\
\hline $2020-2021$ & 60000 \\
\hline TOTAL & $\mathbf{4 5 7 3 0 0}$ \\
\hline
\end{tabular}

Tabla 6. Datos generales de la parroquia Chumblín

Fuente: Convenios de Cooperación Económica firmados con el GAD Parroquial.

Los ejes de inversión como ya se mencionó anteriormente están basados en el Plan de Desarrollo y Ordenamiento Territorial de lo cual se desprende los siguientes porcentajes de inversión:

- $35 \%$ Gestión y servicios comunitarios (infraestructura y logística)

- $30 \%$ Producción

- $15 \%$ Educación y Cultura

- $10 \%$ Fomento del talento (capacitaciones)

- $10 \%$ Salud, seguridad y ambiente

Mediante la dotación de capacitaciones, dotación de insumos e implementos, abonos, semillas, equipo tecnológico, medicina, equipos médicos, contratación de equipo caminero, contratación de docentes se ha beneficiados a más de 15 grupos organizados dentro de la parroquia, entre ellos están:

- Aso. 8 de Septiembre

- Aso. De Ganaderos

- Aso. 4 de Diciembre

- Aso. Vida y Naturaleza 
- Aso. La Natividad

- Aso. De Adultos Mayores

- Más de 10 canales de riego

- U.E.B Víctor Álvarez Torres

- Centro de Salud

- Fortalecimiento de habilidades y saberes de los habitantes a través de las Escuelas de Futbol, de música y danza

- Vialidad e Infraestructura

- Dominio de las TICS /Infocentro Chumblín

- Templo parroquial

Mediante la firma de convenios de cooperación anuales con el Gobierno Local de la Parroquia se ha logrado no solo mejorar las condiciones de vida de los beneficiarios, sino que también el de las familias, hoy en día existen grupos de mujeres cabezas de hogar que se dedican a realizar artesanías, tejidos y también aprovechando las plantas medicinales crean productos, jarabes y entre otros como medicina tradicional.

\section{Modelo teórico para evaluación de impactos}

El modelo que se plantea para la evaluación de impactos, procura solo ser una guía general teórica y didáctica, para una aplicación al momento de realizar la evaluación de un impacto posible.

Evaluar un impacto significa medir los cambios generados en las variables por una intervención en diferentes ámbitos, estos pueden ser social, económico, ambiental, etc.

Para realizar la evaluación de impacto considerar lo siguiente ${ }^{5}$ :

La relación de causalidad: Conocer los cambios que se producen tras un programa o política específica -a nivel social, de la empresa o en los participantes de las mismas e identificar en qué medida estos cambios (efecto) son atribuibles al programa (causa).

La variedad de impactos que puede tener una intervención: -económicos, técnicos, socio-culturales, ambientales- pueden ser previstos (definidos en los objetivos de la actuación a evaluar) o no previstos.

Los distintos impactos: en las personas, las empresas y en la sociedad.

\section{Etapas de evaluación de impacto}

Se establece 4 etapas de estándares abiertos que permiten orientar la evaluación de impactos, estas son:

Etapa 1. Conceptualización: Consiste en la identificación de actores, determinando el o los escenarios de referencia, con el fin de poder definir los elementos orientadores de la evaluación. En esta etapa se deberá precisar:

\footnotetext{
${ }^{5}$ Guía para la evaluación de impactos: http://guia.oitcinterfor.org/conceptualizacion/que-seentiendeevaluacion-impacto
} 
- El alcance: Es el área del proyecto de influencia.

- La visión: Unifica las aspiraciones de los actores del proyecto

- Los objetos focales: Asuntos sobre los cuales se deberán construir estrategias, son el foco de atención del proyecto.

- El diagrama de problemas: El contexto que afecta los asuntos focales, son las relaciones causales entre diversos factores directos e indirectos.

Etapa 2. Planificación de acciones y monitoreo: En esta etapa se deberá desarrollar los objetivos, estrategias, supuestos, metas, revisión de cadenas de resultados y su refinamiento.

Con este modelo conceptual construido será más fácil determinar las variables que pueden verse afectadas por algún posible impacto. A continuación, se ha elaborado una tabla de identificación de variables:

\begin{tabular}{ccc}
\hline & IDENTIFICACIÓN DE VARIABLES \\
\hline MEDIO & TIPO & ESPECIFIDAD \\
\hline Aire & Calidad del aire & Ruidos \\
Agua & Agua superficial \\
Agua subterránea & Geología \\
& & Edafología \\
Medio perceptual & Tierra & Erosión \\
& Vegetación & Procesos estabilidad \\
& Plora y Fauna & Vegetación \\
& Paisaje & Flora y Fauna terrestre \\
& Economía & Calidad paisajística \\
Medio socio- & Empleo \\
& Infraestructura & Actividad económica \\
& & Comunicaciones \\
& Usos del territorio & Planeamiento urbanístico \\
& Patrimonio arqueológico & Ganadería \\
& Población & Afección al patrimonio \\
& Calidad de vida
\end{tabular}

Tabla 7. Identificación de variables según medio, tipo y especificidad

Fuente: Universidad Andina Simón Bolívar

Las variables que pueden ser afectadas por algún tipo de impacto se plantea analizar el medio, tipo y especificidad, a la hora de realizar una evaluación el evaluador podrá escoger o aumentar cualquier variable que se considere necesaria.

Etapa 3. Implementar acciones y monitoreo: En esta etapa se elaborará el plan de monitoreo en donde debe estar identificado los indicadores de monitoreo una estructura básica de evaluación esto permitirá definir el presupuesto necesario y controlar el avance de la ejecución de la evaluación. 
El plan de monitoreo propuesto deberá clarificar los supuestos de cómo una estrategia va a alcanzar los resultados esperados, y de esta manera contribuir a medir los impactos generados.

Mínimamente un plan de monitoreo constará de lo siguiente:

\begin{tabular}{|c|c|}
\hline Objetivos & Se describirá el propósito a alcanzar con la aplicación de la evaluación \\
\hline Estrategias & $\begin{array}{l}\text { Definir las acciones que van a ejecutarse 'para cumplir con los } \\
\text { objetivos. }\end{array}$ \\
\hline Indicadores & $\begin{array}{l}\text { Definir los indicadores suficientes para realizar la evaluación de } \\
\text { impactos. }\end{array}$ \\
\hline Descripción del Indicador & $\begin{array}{l}\text { Se detallará minuciosamente el indicador con el fin de eliminar } \\
\text { subjetividades. }\end{array}$ \\
\hline Unidad de medida & Se establecerá la unidad de medida del indicador \\
\hline Patrón & Se define el comportamiento del indicador. \\
\hline Fónmule & $\begin{array}{l}\text { Seleccionar el método de estimación que más se adapte a la evaluación. } \\
\text { Porcentajes: Razón entre dos variables con una misma unidad de } \\
\text { medida }\end{array}$ \\
\hline rormura & $\begin{array}{l}\text { Tasa de variación: Razón entre una misma variable, pero en periodos } \\
\text { diferentes }\end{array}$ \\
\hline & Promedio: Relación entre dos variables con distinta unidad de medida \\
\hline Frecuencia & Mensual, anual, semestral, trimestral, bimensual. \\
\hline Línea base & $\begin{array}{l}\text { Sirve para conocer el valor de los indicadores al inicio de las } \\
\text { acciones planificadas }\end{array}$ \\
\hline Fuente de información & Determinar el origen de los datos \\
\hline Metas & Se deberá establecer el resultado deseado \\
\hline Supuestos & Datos a ser asumidos como ciertos \\
\hline Costos & Se establecerá el valor monetario de las acciones a realizarse \\
\hline Cronograma de ejecución & $\begin{array}{l}\text { Se construirá un cronograma en detalle que permita la optimización } \\
\text { del tiempo }\end{array}$ \\
\hline Responsable & $\begin{array}{l}\text { Se recomienda nombrar una persona responsable del manejo de la } \\
\text { información }\end{array}$ \\
\hline Observaciones & Aquí se podrá detallar cualquier novedad de la ejecución del plan. \\
\hline
\end{tabular}

Etapa 4. Analizar los resultados: Etapa en donde se deberá preparar los datos para el análisis de los resultados obtenidos, mismos que deben quedar documentados para que sirva de base para una próxima evaluación de impacto.

En base a la matriz de la etapa 3 se deberá procesar y analizar los resultados, con el fin de determinar el tipo de impacto o variación existente en las variables definidas para el estudio.

Los resultados que arrojen el análisis deberán ser entregados a los interesados y responsables, quienes se encargarán de divulgar y preparar las acciones de remedición de los impactos negativos determinados.

Los productos que genera una evaluación son un informe de la evaluación de impacto y una serie de notas de política económica que resumen los resultados fundamentales.

Se plantea la siguiente estructura para la presentación de la estructura del informe del plan de evaluación de impacto: 


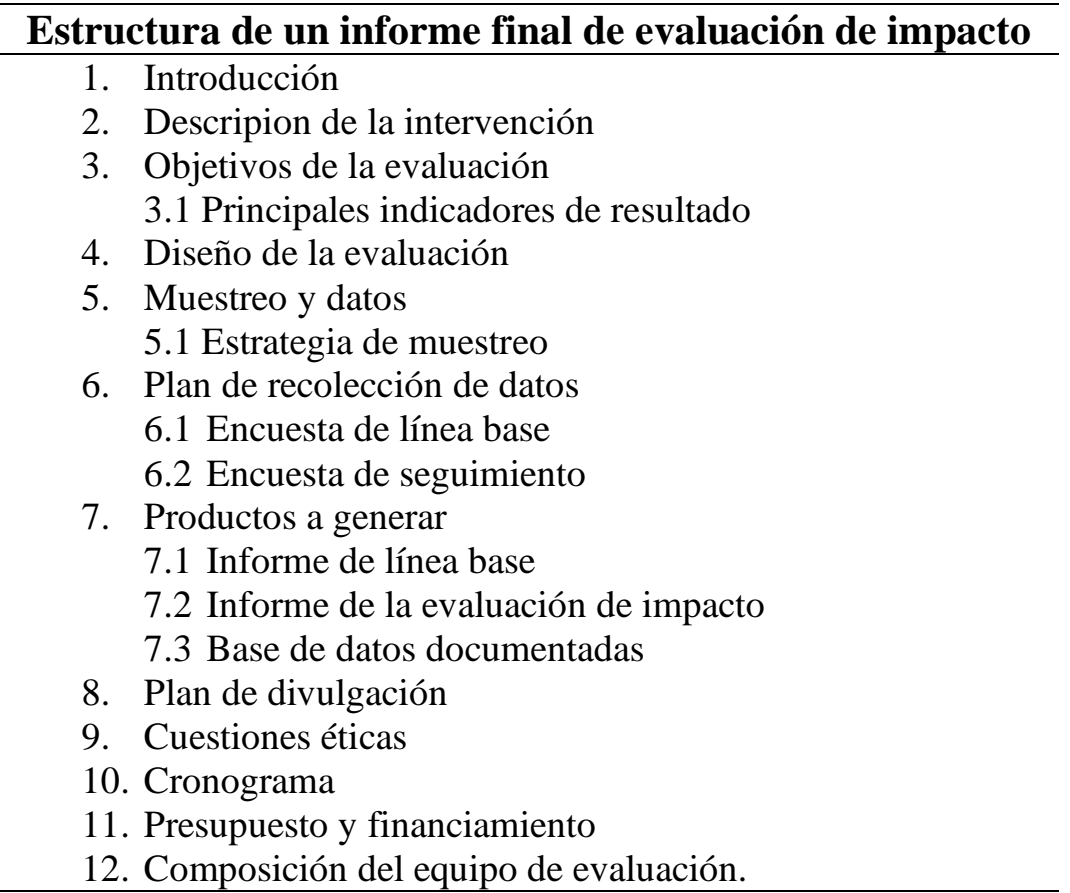

Tabla 9. Estructura de un informe final de evaluación de impacto Fuente: Universidad Simón Bolívar

\section{Conclusiones.}

- En el Ecuador se tiene vestigios de que la minería se originó desde las primeras culturas en diferentes zonas geográficas del país, al inicio de manera artesanal, para luego transformándose en una minería pequeña, mediana y hoy en día a gran escala. Hasta el año 2008, la minería no tenía mayor importancia, es a partir del mandato elaborado por la Asamblea Constituyente que se le dio un orden a esta actividad, señalando procedimientos para su operación y obtención de concesiones, lo que ha permitido tener un mejor control de la minería en el país.

- Por la escasez próxima de los yacimientos petroleros y la abrupta caída del precio del petróleo, el sector minero ha sido visto como la nueva opción para el desarrollo del país; sin embargo, aún son considerados como no compatibles con el buen vivir que es el paradigma base del modelo de desarrollo del país y que implica obtener los recursos que satisfagan las necesidades de sus habitantes sin comprometer los recursos naturales.

- El desarrollo de las políticas generó diferentes perspectivas sobre el uso y control de los recursos naturales: el agua y el suelo. Desde lo oficial y hegemónico se justifica su manejo a nombre del desarrollo y así lo refleja los diferentes indicadores macros de la realidad nacional. Frente a las perspectivas y realidades de los pueblos, en donde se asientan los proyectos estratégicos, son invisibilidades y subordinadas sus ontologías y epistemologías, de entendimiento de su realidad. Está claro que esta otra parte no se ha desarrollado desde la investigación oficial, ya que no se le considera como actor social sino como objeto a ser moldeable a los intereses y necesidades del desarrollo nacional. 
- La actividad minera ha incidido en el desarrollo comunitario de la parroquia Chumblín, pues mediante la firma de convenios de cooperación económica anuales con el Gobierno local se han desarrollados proyectos a favor de los diferentes grupos etareos de la parroquia, la inversión ejecutada ha servido para el beneficio económico y social de la gente. Se puede también manifestar que dentro de la organización parroquial se ha dados lugar a dos frentes opuestos.

- Pese a que los convenios de cooperación económica entre la Empresa INV Minerales y el Gobierno Local son ejecutados cubriendo necesidades de los tres Recintos existentes dentro de la parroquia, canales de riego, grupos organizados, asociaciones y más grupos existentes hay dos sectores opuestos basado principalmente en la ideología de los habitantes y que de una u otra manera generan un colapso interno y de cierta manera no se permite que haya un desarrollo local considerando que desarrollo implica mejorar las condiciones de vida del ser humano. Un gran numero de habitantes de la población chumblinence no acepta la ejecución del proyecto minero Loma Larga, sin embargo, es aceptada al ser beneficiados dentro de diferentes proyectos ejecutados por el GAD Parroquial bajo convenio firmado entre las partes.

- Los principios de responsabilidad social de la empresa hacia la parroquia Chumblín han sido manejados bajo convenios de cooperación firmado con el GAD Parroquial, en los últimos años el eje en donde existe una mayor inversión ha sido en la producción y fomento del talento humano. Así mismo, el compromiso de la empresa minera en lo que se refiere a la responsabilidad del manejo ambiental y técnica, hoy en día, se mantiene bajo los parámetros legales y ambientales exigidos por el Ministerio del Ambiente. Por lo tanto, se puede considerar que, si los trabajos de la empresa minera se mantienen con transparencia y responsabilidad moral hacia la población de la parroquia Chumblín tanto en sus actuales y futuros proyectos, esta influencia es positiva. No obstante, es necesario trabajar arduamente tanto por parte del Gobierno Local y de INV Metals en fomentar la organización parroquial y desde allí en conjunto podrán resolver este problema teniendo en cuenta el desarrollo de toda la parroquia en todo ambito.

\section{Referencias bibliográficas.}

Bustamante, Teodoro y Rommel Lara (compiladores), 2010, El Dorado o la caja de Pandora: matices para pensar la minería en Ecuador, Quito, Flacso.

Chapa, Priscila \& Lopez, Lucia, 2016, Influencia del Proyecto Minero Loma Larga Anterior Quimsacocha- en el Buen Vivir de la Parroquia Rural de San Gerardo (1998 - 2015), Universidad de Cuenca.

Diario El Mercurio. Quimsacocha, un tesori en medio del debate. 2011. Disponible en: http://www.elmercurio.com.ec/299216-quimsacocha-un-tesoro-en-medio-deldebate 2/\#.Vh6NFCtRL_o Publicado: 22/09/2011. 
Gudynas, Eduardo, 2009, El mandato ecológico. Derechos de la Naturaleza y políticas ambientales en la nueva Constitución Abya-Yala, Quito.

Inv Metals. (s.f.). Desarrollo y Exploración en Ecuador. https://sp.invmetals.com/lomalarga-location-and-history/

Inv Metals Inc. 2019. Ni 43-101 Feasibility Study Technical Report. https://sp.invmetals.com/wpcontent/uploads/2019/01/TR_LomaLarga_Jan14_20 19.pdf

Kuhn, Rosie, 2015, No Todo Lo Que Brilla Es Oro conflictos socio ambientales alrededor de dos proyectos de minería a gran escala en el Ecuador, Universidad Andina Simón Bolívar, Quito.

Ley de Mineria, 2009, Registro Oficial Suplemento 517, Quito.

Ley de Mineria, 2011, Registro Oficial Suplemento 583, Quito.

Mora, Gabriela, 2015, Análisis de la Inversión Directa proveniente de Canadá y su incidencia en la Economía Ecuatoriana en el Sector de la Minería durante el Período 2008 - 2012, Universidad de Cuenca.

Ordeñana Rodriguez, X., \& Dominguez, J. M. (s.f.). Beneficios y amenazas de la minería a Gran Escala en Ecuador. Obtenido de Energía y Minas: http://www.espae.espol.edu.ec/images/FTP/beneficiosyamenazasdelamineria.pdf

Pillajo, Edgar. 2011. La minería al servicio de los ecuatorianos. Fungeomine, Universidad Central del Ecuador, Quito

Plan Nacional de Desarrollo del Sector Minero 2020-2030. Ministerio de Energia y Recursos Naturales no Renovables, Viceministerio de Minas.

Redacción Comercial. Proyecto minero Loma Larga: compatible con el medio ambiente. https://www.primicias.ec/noticias/patrocinado/proyecto-minero-loma-largacompatible con-el-ambiente/Primicias.

Sacher, William y Alberto Acosta, 2012, La Minería en Gran Escala en Ecuador. Quito, Abya Yala.

Vega, Gabriela, 2018. Análisis de la Inversión Extranjera Directa en Ecuador y su Incidencia en la Extracción Minera Metálica en el Periodo 2007-2014. Universidad Católica del Ecuador.

Zeballos, E.J. and S. Garry, 2010, “An Overview of Employment Trends and Working Conditions by Economic Activity in Jobs Recovery" (Washington, Sectorial Activities Department, International Labour Organization (ILO), 42- 50

\section{【| Ciencia}




\section{PARA CITAR EL ARTÍCULO INDEXADO.}

Chuñir Panjón, L., Covri Rivera, D., \& Castillo Ortega, Y. (2021). Incidencia de la actividad minera en el desarrollo social de la parroquia Chumblín del cantón San Fernando. ConcienciaDigital, 4(1.2),

422-445. https://doi.org/10.33262/concienciadigital.v4i1.2.1608

\section{Ciencia \\ LDigital}

El artículo que se publica es de exclusiva responsabilidad de los autores y no necesariamente reflejan el pensamiento de la Revista Conciencia Digital.

El artículo queda en propiedad de la revista y, por tanto, su publicación parcial y/o total en otro medio tiene que ser autorizado por el director de la Revista Conciencia Digital.

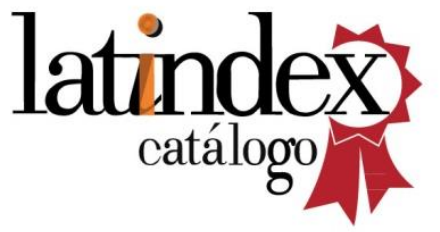

